\title{
Constructing Choquet integral-based operators that generalize weighted means and OWA operators
}

\author{
Bonifacio Llamazares* \\ Departamento de Economía Aplicada, Instituto de Matemáticas (IMUVA), Universidad de Valladolid, Avda. Valle de \\ Esgueva 6, 47011 Valladolid, Spain.
}

\begin{abstract}
In this paper we introduce the semi-uninorm based ordered weighted averaging (SUOWA) operators, a new class of aggregation functions that, as WOWA operators, simultaneously generalize weighted means and OWA operators. To do this we take into account that weighted means and OWA operators are particular cases of Choquet integral. So, SUOWA operators are Choquet integral-based operators where their capacities are constructed by using semi-uninorms and the values of the capacities associated to the weighted means and the OWA operators. We also show some interesting properties of these new operators and provide examples showing that SUOWA and WOWA operators are different classes of aggregation operators.
\end{abstract}

Keywords: Choquet integral, weighted means, OWA operators, SUOWA operators, WOWA operators, semi-uninorms.

\section{Introduction}

Aggregation information is a usual activity in many research fields. Weighted means and ordered weighted averaging (OWA) operators (introduced by Yager [1]) are well-known functions widely used for this task. Both classes of functions are defined by means of weighting vectors, but their behavior is quite different: Weighted means allow to weight each information source in relation to their reliability while OWA operators allow to weight the values according to their ordering.

Some authors (see, among others, Torra [2], Torra and Godo [3, pp. 160-161], Torra and Narukawa [4, pp. 150-151] and Yager and Alajlan [5]) have reported the need for both weightings in several fields such as robotics, vision, fuzzy logic controllers, constraint satisfaction problems, scheduling and decision making. For instance, suppose we have several sensors to measure a physical property. On the one hand, sensors may be of different quality and precision, so a weighted mean type aggregation is necessary. On the other hand, to prevent a faulty sensor alters the measurement, we might consider an OWA type aggregation where the maximum and minimum values

${ }^{*}$ Tel.: +34-983-186544; fax: +34-983-423299.

Email address: boni@eco. uva.es (Bonifacio Llamazares) 
are not taking into account. A similar situation occurs when a committee of experts has to assess several candidates or proposals. On the one hand, a weighted mean type aggregation is suitable for reflecting the expertness or the confidence in the judgment of each expert. On the other hand, an OWA type aggregation allows us to deal with situations where an expert feels an excessive acceptance or rejection towards some of the candidates or proposals.

Different types of models have appeared in the literature to deal with this kind of situations (see, for instance, Yager [6, 7], Calvo and Mesiar [8] and Yager and Alajlan [5]). Some families of functions suggested in the literature generalize weighted means and OWA operators in the sense that one of these functions is obtained when the other one has a "neutral" behavior; that is, its weighting vector is that of the arithmetic mean. This is the case of the operator proposed by Engemann et al. [9], the weighted OWA (WOWA) operator (Torra [2]), the hybrid weighted averaging (HWA) operator (Xu and Da [10]), the IP-OWA operator (Merigó [11]) and the hybrid weighted arithmetical averaging (HWAA) operator (Lin and Jiang [12]). Notice that, as pointed out by Wang [13], the IP-OWA operator and the HWAA operator are the same type of aggregation functions, although the interpretation of their weighting vectors are different. In fact, these functions coincide with the operator proposed by Engemann et al. [9], which has been analyzed by Llamazares [14] (on the monotonicity of this operator, see also Liu [15], Wang [13] and Lin [16]).

The aim of this work is to introduce new aggregation operators that generalize the weighted means and the OWA operators in the above sense. To do this we take into account that weighted means and OWA operators are particular cases of Choquet integral. So, SUOWA operators are Choquet integral-based operators where their capacities are constructed by using semi-uninorms and the values of the capacities associated to the weighted means and the OWA operators. We also establish some interesting properties of these new operators and show that, in the case of using idempotent semi-uninorms, the smallest and the largest one are obtained by using two well-known uninorms given by Yager and Rybalov [17].

The paper is organized as follows. In Section 2 we recall some properties of aggregation functions and some basic concepts on weighted means, OWA operators, Choquet integral, uninorms and semi-uninorms. Section 3 is devoted to the construction of capacities by means of which we can generalize weighted means and OWA operators. In Section 4 we formally define SUOWA operators and show some properties of this class of functions. Some examples are given in Section 5 to illustrate the construction of games and capacities by using semi-uninorms and the fact that WOWA and SUOWA operators are different classes of aggregation operators. Finally, some concluding remarks are provided in Section 6.

\section{Aggregation functions}

Throughout the paper we will use the following notation: $N=\{1, \ldots, n\}$; given $A \subseteq N,|A|$ will denote the cardinality of $A$; vectors will be denoted in bold; $\boldsymbol{\eta}$ will denote the vector $(1 / n, \ldots, 1 / n)$; $\boldsymbol{x} \geq \boldsymbol{y}$ will mean $x_{i} \geq y_{i}$ for all $i \in N$; given $\boldsymbol{x} \in \mathbb{R}^{n}$, let $x_{[1]} \geq \cdots \geq x_{[n]}$ will denote the components of $\boldsymbol{x}$ in decreasing order, and let $x_{(1)} \leq \cdots \leq x_{(n)}$ will denote the components of $\boldsymbol{x}$ in increasing order.

In the following definition we present some well-known properties usually demanded to the functions used in the aggregation processes. 
Definition 1. Let $F: \mathbb{R}^{n} \longrightarrow \mathbb{R}$ be a function.

1. $F$ is symmetric if $F\left(x_{\sigma(1)}, \ldots, x_{\sigma(n)}\right)=F\left(x_{1}, \ldots, x_{n}\right)$ for all $\boldsymbol{x} \in \mathbb{R}^{n}$ and for all permutation $\sigma$ of $N$.

2. $F$ is monotonic if $\boldsymbol{x} \geq \boldsymbol{y}$ implies $F(\boldsymbol{x}) \geq F(\boldsymbol{y})$ for all $\boldsymbol{x}, \boldsymbol{y} \in \mathbb{R}^{n}$.

3. $F$ is idempotent if $F(x, \ldots, x)=x$ for all $x \in \mathbb{R}$.

4. $F$ is compensative (or internal) if $\min (\boldsymbol{x}) \leq F(\boldsymbol{x}) \leq \max (\boldsymbol{x})$ for all $\boldsymbol{x} \in \mathbb{R}^{n}$.

5. $F$ is homogeneous of degree 1 (or ratio scale invariant) if $F(\lambda \boldsymbol{x})=\lambda F(\boldsymbol{x})$ for all $\boldsymbol{x} \in \mathbb{R}^{n}$ and for all $\lambda>0$.

\subsection{Weighted means and OWA operators}

Weighted means and OWA operators are defined by vectors with non-negative components whose sum is 1 .

Definition 2. A vector $\boldsymbol{q} \in \mathbb{R}^{n}$ is a weighting vector if $\boldsymbol{q} \in[0,1]^{n}$ and $\sum_{i=1}^{n} q_{i}=1$.

Definition 3. Let $\boldsymbol{p}$ be a weighting vector. The weighted mean associated to $\boldsymbol{p}$ is the function $M_{\boldsymbol{p}}: \mathbb{R}^{n} \longrightarrow \mathbb{R}$ given by

$$
M_{\boldsymbol{p}}(\boldsymbol{x})=\sum_{i=1}^{n} p_{i} x_{i}
$$

Weighted means are monotonic, idempotent, compensative and homogeneous of degree 1 functions. For its part, OWA operators, introduced by Yager [1], are defined in a similar way to the weighted means, but with the values of the variables previously ordered in a decreasing way. Therefore they are symmetric, and also satisfy other interesting properties such as monotonicity, idempotence, compensativeness and homogeneity of degree 1.

Definition 4. Let $\boldsymbol{w}$ be a weighting vector. The OWA operator associated to $\boldsymbol{w}$ is the function $O_{w}: \mathbb{R}^{n} \longrightarrow \mathbb{R}$ given by

$$
O_{w}(\boldsymbol{x})=\sum_{i=1}^{n} w_{i} x_{[i]} .
$$

\subsection{Choquet integral}

Choquet integral-based operators are a very important class of aggregation operators which have had a wide variety of applications in the last years (see, for instance, Grabisch et al. [18] and Grabisch and Labreuche [19]). Before introducing the Choquet integral we recall the notions of game and capacity (see also fuzzy measures in Sugeno [20]).

\section{Definition 5.}

1. A game $v$ on $N$ is a set function, $v: 2^{N} \longrightarrow \mathbb{R}$ satisfying $v(\varnothing)=0$.

2. A capacity (or fuzzy measure) $\mu$ on $N$ is a game on $N$ satisfying $\mu(A) \leq \mu(B)$ whenever $A \subseteq B$. In particular, it follows that $\mu: 2^{N} \longrightarrow[0, \infty)$. A capacity $\mu$ is said to be normalized if $\mu(N)=1$. 
The Choquet integral can be defined in a general context (see Choquet [21], Murofushi and Sugeno [22] and Denneberg [23]). However, we only consider the Choquet integral in the framework that we are dealing with here (see Grabisch et al. [24, p. 181]).

Definition 6. Let $\mu$ be a capacity on $N$. The Choquet integral with respect to $\mu$ is the function $C_{\mu}: \mathbb{R}^{n} \longrightarrow \mathbb{R}$ given by

$$
C_{\mu}(\boldsymbol{x})=\sum_{i=1}^{n} \mu\left(B_{(i)}\right)\left(x_{(i)}-x_{(i-1)}\right),
$$

where $B_{(i)}=\{(i), \ldots,(n)\}$ and, by convention, $x_{(0)}=0$.

Notice that the Choquet integral can be defined with respect to games instead of capacities (see again Grabisch et al. [24, p. 181]). In this case, the Choquet integral satisfies the following properties (Grabisch et al. [24, p. 193 and p. 196]):

Remark 1. If $v$ is a game on $N$ and $C_{v}$ is the Choquet integral with respect to $v$, then

1. $C_{v}$ is continuous.

2. $C_{v}$ is homogeneous of degree 1 .

3. $C_{v}$ is monotonic if and only if $v$ is a capacity.

4. $C_{v}$ is idempotent when $v(N)=1$.

5. $C_{v}$ is compensative when $v$ is a normalized capacity.

In analogy with OWA operators, in the sequel we show an equivalent representation of Choquet integral by means of decreasing sequences of values (see also Torra [25]). Given $\boldsymbol{x} \in \mathbb{R}^{n}$, we can consider $(\cdot)$ and $[\cdot]$ so that $(i)=[n+1-i]$ for all $i \in N$. In this case,

$$
B_{(i)}=\{(i), \ldots,(n)\}=\{[1], \ldots,[n+1-i]\},
$$

and this set will be denoted by $A_{[n+1-i]}$. Consequently,

$$
\begin{aligned}
C_{\mu}(\boldsymbol{x}) & =\mu\left(B_{(1)}\right) x_{(1)}+\sum_{j=2}^{n} \mu\left(B_{(j)}\right)\left(x_{(j)}-x_{(j-1)}\right) \\
& =\mu\left(A_{[n]}\right) x_{[n]}+\sum_{j=2}^{n} \mu\left(A_{[n+1-j]}\right)\left(x_{[n+1-j]}-x_{[n+2-j]}\right) \\
& =\mu\left(A_{[n]}\right) x_{[n]}+\sum_{i=1}^{n-1} \mu\left(A_{[i]}\right)\left(x_{[i]}-x_{[i+1]}\right) \\
& =\sum_{i=1}^{n} \mu\left(A_{[i]}\right)\left(x_{[i]}-x_{[i+1]}\right),
\end{aligned}
$$

where $A_{[i]}=\{[1], \ldots,[i]\}$ and, by convention, $x_{[n+1]}=0$.

From the previous expression, it is straightforward to check that the Choquet integral can be written as

$$
C_{\mu}(\boldsymbol{x})=\sum_{i=1}^{n}\left(\mu\left(A_{[i]}\right)-\mu\left(A_{[i-1]}\right)\right) x_{[i]},
$$


with the convention $A_{[0]}=\varnothing$. From this formula we can easily see that weighted means and OWA operators are a special type of Choquet integral (see also Fodor et al. [26] and Grabisch [27, 28]). Remark 2. Let $\mu$ be a capacity on $N$.

1. $C_{\mu}$ is the weighted mean $M_{p}$ if $\mu\left(A_{[i]}\right)-\mu\left(A_{[i-1]}\right)=p_{[i]}$ for all $i \in N$, or, equivalently, $\mu\left(A_{[i]}\right)=\sum_{j=1}^{i} p_{[j]}$ for all $i \in N$. Therefore $\mu(A)=\sum_{i \in A} p_{i}$ for any nonempty subset $A$ of $N$.

2. $C_{\mu}$ is the OWA operator $O_{w}$ if $\mu\left(A_{[i]}\right)-\mu\left(A_{[i-1]}\right)=w_{i}$ for all $i \in N$, or, equivalently, $\mu\left(A_{[i]}\right)=$ $\sum_{j=1}^{i} w_{j}$ for all $i \in N$. Therefore $\mu(A)=\sum_{i=1}^{|A|} w_{i}$ for any nonempty subset $A$ of $N$.

\subsection{Uninorms and semi-uninorms}

Uninorms were introduced by Yager and Rybalov [17] as a generalization of t-norms and tconorms (see also Fodor et al. [29], and Fodor and De Baets [30]).

Definition 7. A function $U:[0,1]^{2} \rightarrow[0,1]$ is a uninorm if it is symmetric, associative $(U(x, U(y, z))=U(U(x, y), z)$ for all $x, y, z \in[0,1])$, monotonic and possesses a neutral element $e \in[0,1](U(e, x)=U(x, e)=x$ for all $x \in[0,1])$.

For its part, semi-uninorms were proposed by Liu [31] as a generalization of uninorms by dispensing with the symmetry and associativity properties.

Definition 8. A function $U:[0,1]^{2} \longrightarrow[0,1]$ is a semi-uninorm if it is monotonic and possesses a neutral element $e \in[0,1]$.

The set of semi-uninorms with neutral element $e \in[0,1]$ will be denoted by $\mathcal{U}^{e}$. Semiuninorms satisfy the following boundary conditions: $U(0,0)=0$ and $U(1,1)=1$. Moreover, the structure of semi-uninorms has been studied by Liu [31, Proposition 2.1], and it is showed in Figure 1.

Proposition 1. Let $U \in \mathcal{U}^{e}$. Then:

1. $U(x, y)=\min (x, y)$ if $(x, y) \in\{e\} \times[0, e] \cup[0, e) \times\{e\}$.

2. $U(x, y)=\max (x, y)$ if $(x, y) \in\{e\} \times(e, 1] \cup(e, 1] \times\{e\}$.

3. $0 \leq U(x, y) \leq \min (x, y)$ if $(x, y) \in[0, e)^{2}$.

4. $\max (x, y) \leq U(x, y) \leq 1$ if $(x, y) \in(e, 1]^{2}$.

5. $\min (x, y) \leq U(x, y) \leq \max (x, y)$ if $(x, y) \in[0, e) \times(e, 1] \cup(e, 1] \times[0, e)$.

Idempotent semi-uninorms play an important role in our generalization of weighted means and OWA operators. A characterization of idempotent semi-uninorms can be straightforward obtained from Liu [31, Proposition 2.2] (see also De Baets [32] for a characterization of idempotent uninorms), and their structure is shown in Figure 2.

Proposition 2. Let $U \in \mathcal{U}^{e}$. $U$ is idempotent if and only if

$$
U(x, y)= \begin{cases}\min (x, y) & \text { if }(x, y) \in[0, e]^{2}, \\ \max (x, y) & \text { if }(x, y) \in[e, 1]^{2} \backslash\{(e, e)\}, \\ P(x, y) & \text { otherwise, }\end{cases}
$$

where $P:[0, e) \times(e, 1] \cup(e, 1] \times[0, e) \longrightarrow[0,1]$ is monotonic and $\min (x, y) \leq P(x, y) \leq \max (x, y)$ for all $(x, y) \in[0, e) \times(e, 1] \cup(e, 1] \times[0, e)$. 


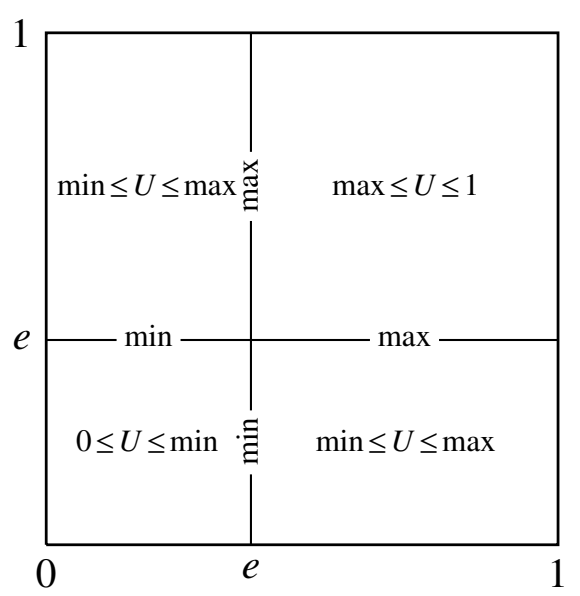

Figure 1: The structure of semi-uninorms.

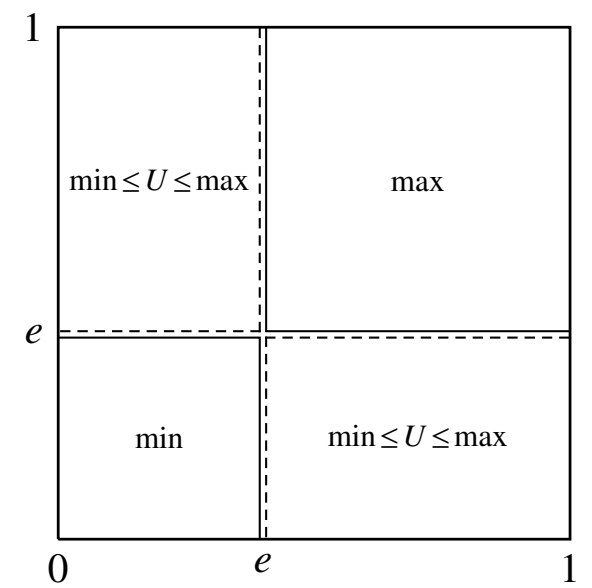

Figure 2: The structure of idempotent semi-uninorms. 
The set of idempotent semi-uninorms with neutral element $e \in[0,1]$ will be denoted by $\mathcal{U}_{\mathrm{i}}^{e}$. Notice that the smallest and the largest idempotent semi-uninorm are, respectively, the following uninorms (which were given by Yager and Rybalov [17]):

$$
U_{\min }(x, y)= \begin{cases}\max (x, y) & \text { if }(x, y) \in[e, 1]^{2}, \\ \min (x, y) & \text { otherwise }\end{cases}
$$

and

$$
U_{\max }(x, y)= \begin{cases}\min (x, y) & \text { if }(x, y) \in[0, e]^{2} \\ \max (x, y) & \text { otherwise }\end{cases}
$$

\section{Capacities}

Given two weighting vectors $\boldsymbol{p}$ and $\boldsymbol{w}$, our aim is to find new functions based on the Choquet integral, $F_{\boldsymbol{p}, \boldsymbol{w}}$, that, as WOWA operators (see Torra [2]), simultaneously generalize weighted means and OWA operators in the following sense: $F_{p, \eta}=M_{p}$ and $F_{\eta, w}=O_{w}$.

According to (1), if we represent the function we are looking for as

$$
F_{p, w}(\boldsymbol{x})=\sum_{i=1}^{n}\left(\mu_{p, w}\left(A_{[i]}\right)-\mu_{p, w}\left(A_{[i-1]}\right)\right) x_{[i]},
$$

with $A_{[0]}=\varnothing$, then, by Remark $2, F_{p, w}$ simultaneously generalize weighted means and OWA operators when for any nonempty subset $A$ of $N$ the capacity $\mu_{p, w}$ satisfies

$$
\mu_{\boldsymbol{p}, \boldsymbol{\eta}}(A)=\sum_{i \in A} p_{i}, \quad \mu_{\boldsymbol{\eta}, \boldsymbol{w}}(A)=\sum_{i=1}^{|A|} w_{i} .
$$

By Remark 1, we are interested in using normalized capacities so that the function $F_{p, w}$ has desirable properties. However, it is not straightforward to get normalized capacities satisfying the conditions given by (2). To solve this problem, in the next subsection we introduce the notion of monotonic cover of a game (see Maschler and Peleg [33] and Maschler et al. [34]).

\subsection{Monotonic cover of a game}

An easy way to obtain a capacity $\hat{v}$ from a game $v$ is to consider the monotonic cover of the game: For each nonempty subset $A$ of $N$, we take the maximum value of the set function over the subsets contained in $A$.

Definition 9. Let $v$ be a game on $N$. The monotonic cover of $v$ is the set function $\hat{v}: 2^{N} \longrightarrow[0, \infty)$ given by $\hat{v}(\varnothing)=0$ and

$$
\hat{v}(A)=\max _{B \subseteq A} v(B)
$$

for any nonempty subset $A$ of $N$. 
It is worth noting that the monotonic cover of a game $v$ is the least capacity satisfying $v(A) \leq$ $\hat{v}(A)$ for all $A \subseteq N$. Moreover, to determine $\hat{v}$ from a practical point of view, it is possible to recursively define $\hat{v}$ taking into account the subsets contained in $A$ with one less element; that is, for each nonempty subset $A$ of $N$,

$$
\begin{aligned}
\hat{v}(A) & =\max _{B \subseteq A} v(B)=\max \left(v(A), \max _{B \subseteq A} v(B)\right) \\
& =\max \left(v(A), \max _{i \in A} \hat{v}(A \backslash\{i\})\right) .
\end{aligned}
$$

Some basic properties of $\hat{v}$ are given in the sequel.

Remark 3. Let $v$ be a game on $N$. Then:

1. If $v$ is a capacity, then $\hat{v}=v$.

2. If $v(A) \leq 1$ for all $A \subseteq N$ and $v(N)=1$, then $\hat{v}$ is a normalized capacity.

The usual order relation between games is preserved in the corresponding monotonic covers.

Proposition 3. Let $v_{1}$ and $v_{2}$ be two games on $N$. If $v_{1}(A) \leq v_{2}(A)$ for all $A \subseteq N$, then $\hat{v}_{1}(A) \leq$ $\hat{v}_{2}(A)$ for all $A \subseteq N$.

Proof. Given a nonempty subset $A$ of $N$,

$$
\hat{v}_{1}(A)=\max _{B \subseteq A} v_{1}(B) \leq \max _{B \subseteq A} v_{2}(B)=\hat{v}_{2}(A) .
$$

In addition to the previous properties, it is worth noting that if a game on $N$ fulfils the conditions given by (2), then the monotonic cover of the game also satisfies these conditions.

Proposition 4. Let $\boldsymbol{p}$ and $\boldsymbol{w}$ be two weighting vectors and let $v_{p, w}$ be a game on $N$ such that

$$
v_{\boldsymbol{p}, \boldsymbol{\eta}}(A)=\sum_{i \in A} p_{i} \quad \text { and } \quad v_{\boldsymbol{\eta}, \boldsymbol{w}}(A)=\sum_{i=1}^{|A|} w_{i}
$$

for any nonempty subset $A$ of $N$. Then,

$$
\hat{v}_{p, \boldsymbol{\eta}}(A)=\sum_{i \in A} p_{i} \quad \text { and } \quad \hat{v}_{\boldsymbol{\eta}, \boldsymbol{w}}(A)=\sum_{i=1}^{|A|} w_{i}
$$

for any nonempty subset $A$ of $N$.

Proof. Given a nonempty subset $A$ of $N$,

$$
\begin{aligned}
& \hat{v}_{\boldsymbol{p}, \boldsymbol{\eta}}(A)=\max _{B \subseteq A} v_{\boldsymbol{p}, \boldsymbol{\eta}}(B)=\max _{B \subseteq A} \sum_{i \in B} p_{i}=\sum_{i \in A} p_{i}, \\
& \hat{v}_{\boldsymbol{\eta}, \boldsymbol{w}}(A)=\max _{B \subseteq A} v_{\boldsymbol{\eta}, \boldsymbol{w}}(B)=\max _{B \subseteq A} \sum_{i=1}^{|B|} w_{i}=\sum_{i=1}^{|A|} w_{i} .
\end{aligned}
$$




\subsection{Constructing capacities by using semi-uninorms}

Given a nonempty subset $A$ of $N$, weighted means and OWA operators are generated through normalized capacities defined by the values $\sum_{i \in A} p_{i}$ and $\sum_{i=1}^{|A|} w_{i}$, respectively. Therefore, our first intention is to consider a game on $N$ given as a function of these values; that is,

$$
v_{p, w}^{f}(A)=f\left(\sum_{i \in A} p_{i}, \sum_{i=1}^{|A|} w_{i}\right) .
$$

However, conditions given by (2) imply that, if $|A|=j$, then

$$
v_{\boldsymbol{\eta}, \boldsymbol{w}}^{f}(A)=f\left(\frac{j}{n}, \sum_{i=1}^{|A|} w_{i}\right)=\sum_{i=1}^{|A|} w_{i},
$$

and

$$
v_{p, \boldsymbol{\eta}}^{f}(A)=f\left(\sum_{i \in A} p_{i}, \frac{j}{n}\right)=\sum_{i \in A} p_{i},
$$

that is, $j / n$ should be a neutral element of the function $f$. Since the neutral element of a function is unique, we should use different functions according to the cardinality of the set $A$. To avoid this, we make a transformation of the values $\sum_{i \in A} p_{i}$ and $\sum_{i=1}^{|A|} w_{i}$ taking into account the cardinality of the set $A$. So, when the set $A$ is nonempty, we consider the set function

$$
v_{p, w}^{f}(A)=|A| \cdot f\left(\frac{\sum_{i \in A} p_{i}}{|A|}, \frac{\sum_{i=1}^{|A|} w_{i}}{|A|}\right) .
$$

In this way, conditions given by (2) are satisfied when $f$ is a function with neutral element $1 / n$. When we look for functions with neutral elements, uninorms appear in a natural way. Nevertheless, for our purposes we can consider semi-uninorms by dispensing with the symmetry and associativity properties (although symmetry is very interesting if we want a treatment egalitarian between the weighting vectors $\boldsymbol{p}$ and $\boldsymbol{w}$ ). Taking into account the above considerations, we can now define the game associated to two weighting vectors and a semi-uninorm.

Definition 10. Let $\boldsymbol{p}$ and $\boldsymbol{w}$ be two weighting vectors and let $U \in \mathcal{U}^{1 / n}$. The game associated to $\boldsymbol{p}$, $\boldsymbol{w}$ and $U$ is the set function $v_{p, w}^{U}: 2^{N} \longrightarrow \mathbb{R}$ defined by $v_{p, w}^{U}(\varnothing)=0$ and

$$
v_{p, w}^{U}(A)=|A| \cdot U\left(\frac{\sum_{i \in A} p_{i}}{|A|}, \frac{\sum_{i=1}^{|A|} w_{i}}{|A|}\right)
$$

for any nonempty subset $A$ of $N$. 
It is easy to check that $v_{p, w}^{U}$ satisfies the conditions given by (2) and that $v_{p, w}^{U}(N)=1$. However, the game $v_{p, w}^{U}$ may not be a capacity; that is, it may not be monotonic as we show in the following example.

Example 1. Let $\boldsymbol{p}=(0.5,0.2,0.1,0.1,0.1)$ and $\boldsymbol{w}=(0.6,0.2,0,0,0.2)$. Given $U \in \mathcal{U}^{0.2}$, when $A=\{2\}$ we have

$$
v_{p, w}^{U}(A)=U(0.2,0.6)=0.6 .
$$

However, if $B=\{2,3,4,5\}$, we get

$$
v_{p, w}^{U}(B)=4 U(0.5 / 4,0.2)=0.5 \text {. }
$$

Therefore, $A \subseteq B$ but $v_{p, w}^{U}(A)>v_{p, w}^{U}(B)$; that is, $v_{p, w}^{U}$ is not monotonic.

This shortcoming can be solved by using the monotonic cover of a game.

Definition 11. Let $\boldsymbol{p}$ and $\boldsymbol{w}$ be two weighting vectors, let $U \in \mathcal{U}^{1 / n}$, and let $v_{p, w}^{U}$ be the game associated to $\boldsymbol{p}, \boldsymbol{w}$ and $U$. The monotonic cover of the game $v_{\boldsymbol{p}, \boldsymbol{w}}^{U}, \hat{v}_{\boldsymbol{p}, \boldsymbol{w}}^{U}$, will be called the capacity associated to $\boldsymbol{p}, \boldsymbol{w}$ and $U$.

Notice that, by definition, $v_{p, w}^{U}(A) \geq 0$ for all $A \subseteq N$. Therefore, when $|A|=1$ we have $\hat{v}_{p, w}^{U}(A)=v_{p, w}^{U}(A)$.

Once we know how to obtain capacities, our next goal is to get normalized capacities. According to (2) of Remark 3 and Proposition 4, in order to obtain a normalized capacity on $N$ satisfying the conditions given by (2) it is sufficient to find a game $v_{p, w}$ on $N$ satisfying these conditions and such that $v_{p, w}(A) \leq 1$ for all $A \subseteq N$ and $v_{p, w}(N)=1$. However, the game $v_{p, w}^{U}$ may not satisfy the condition $v_{p, w}^{U}(A) \leq 1$ for all $A \subseteq N$, as we show in the following example.

Example 2. Let $\boldsymbol{p}=(0.5,0.2,0.1,0.1,0.1)$ and $\boldsymbol{w}=(0.6,0.2,0,0,0.2)$. Consider the semiuninorm (see Calvo et al. [35, p. 11]) given by

$$
U(x, y)=\max (0, \min (1, x+y-0.2)) .
$$

It is easy to check that $U \in \mathcal{U}^{0.2}$ and that it can be written as

$$
U(x, y)= \begin{cases}0 & \text { if } x+y \leq 0.2, \\ 1 & \text { if } x+y \geq 1.2 \\ x+y-0.2 & \text { otherwise }\end{cases}
$$

If $A=\{1,2\}$ we have

$$
v_{p, w}^{U}(A)=2 U(0.35,0.4)=2 \cdot 0.55=1.1>1 .
$$

In the following proposition we characterize the semi-uninorms $U$ with neutral element $1 / n$ for which $v_{p, w}^{U}(A) \leq 1$ for all weighting vectors $\boldsymbol{p}$ and $\boldsymbol{w}$, and for all $A \subseteq N$.

Proposition 5. Let $U \in \mathcal{U}^{1 / n}$. The following conditions are equivalent: 
1. $v_{p, w}^{U}(A) \leq 1$ for all weighting vectors $\boldsymbol{p}$ and $\boldsymbol{w}$, and for all $A \subseteq N$.

2. $U(1 / k, 1 / k) \leq 1 / k$ for all $k \in N$.

\section{Proof.}

(1) $\Rightarrow$ (2) : Given $k \in N$, consider the weighting vectors $\boldsymbol{p}=\boldsymbol{w}=(1,0, \ldots, 0)$. When $A=\{1,2, \ldots, k\}$, we get $v_{p, w}^{U}(A)=k U(1 / k, 1 / k)$. Since, by hypothesis, $v_{p, w}^{U}(A) \leq 1$, we have $U(1 / k, 1 / k) \leq 1 / k$.

$(2) \Rightarrow(1)$ : Given $\boldsymbol{p}$ and $\boldsymbol{w}$ two weighting vectors and a nonempty subset $A$ of $N$, we have

$$
v_{p, w}^{U}(A)=|A| U\left(\frac{\sum_{i \in A} p_{i}}{|A|}, \frac{\sum_{i=1}^{|A|} w_{i}}{|A|}\right) \leq|A| U\left(\frac{1}{|A|}, \frac{1}{|A|}\right) \leq|A| \frac{1}{|A|}=1 .
$$

The set of semi-uninorms with neutral element $1 / n$ satisfying the second condition given in the previous proposition will be denoted by $\widetilde{\mathcal{U}}^{1 / n}$; that is,

$$
\widetilde{\mathcal{U}}^{1 / n}=\left\{U \in \mathcal{U}^{1 / n} \mid U(1 / k, 1 / k) \leq 1 / k \text { for all } k \in N\right\} .
$$

It is worth mentioning that the smallest and the largest elements of $\widetilde{\mathcal{U}}^{1 / n}$ are, respectively, the following semi-uninorms:

$$
U_{\perp}(x, y)= \begin{cases}0 & \text { if }(x, y) \in[0,1 / n)^{2} \\ \max (x, y) & \text { if }(x, y) \in[1 / n, 1]^{2} \\ \min (x, y) & \text { otherwise }\end{cases}
$$

and

$$
U_{\mathrm{T}}(x, y)= \begin{cases}\min (x, y) & \text { if }(x, y) \in[0,1 / n]^{2}, \\ 1 / k & \text { if }(x, y) \in(1 / n, 1 / k]^{2} \backslash(1 / n, 1 /(k+1)]^{2}, \quad(k=1, \ldots, n-1), \\ \max (x, y) & \text { otherwise. }\end{cases}
$$

Notice that any idempotent semi-uninorm belongs to $\widetilde{\mathcal{U}}^{1 / n}$. Moreover, as we are going to see in the following, the use of idempotent semi-uninorms allows us to obtain some interesting properties (see also Proposition 7). So, for any nonempty subset $A$ of $N$, both $v_{p, w}^{U}(A)$ and $\hat{v}_{p, w}^{U}(A)$ range between the minimum and the maximum of the values $\sum_{i \in A} p_{i}$ and $\sum_{i=1}^{|A|} w_{i}$ when we consider idempotent semi-uninorms. Therefore, when both values are equal, the values of the game and the capacity coincide with them.

Proposition 6. Let $\boldsymbol{p}$ and $\boldsymbol{w}$ be two weighting vectors, $U \in \mathcal{U}_{\mathrm{i}}^{1 / n}$ and let $v_{\boldsymbol{p}, \boldsymbol{w}}^{U}$ be the game on $N$ associated to $\boldsymbol{p}, \boldsymbol{w}$ and $U$. Then, for any nonempty subset $A$ of $N$, we have

$$
\min \left(\sum_{i \in A} p_{i}, \sum_{i=1}^{|A|} w_{i}\right) \leq v_{p, w}^{U}(A) \leq \max \left(\sum_{i \in A} p_{i}, \sum_{i=1}^{|A|} w_{i}\right) .
$$


Proof. Given a nonempty subset $A$ of $N$,

$$
v_{p, w}^{U}(A)=|A| U\left(\frac{\sum_{i \in A} p_{i}}{|A|}, \frac{\sum_{i=1}^{|A|} w_{i}}{|A|}\right) \geq|A| \min \left(\frac{\sum_{i \in A} p_{i}}{|A|}, \frac{\sum_{i=1}^{|A|} w_{i}}{|A|}\right)=\min \left(\sum_{i \in A} p_{i}, \sum_{i=1}^{|A|} w_{i}\right) .
$$

The second inequality is obtained in a similar way.

Corollary 1. Let $\boldsymbol{p}$ and $\boldsymbol{w}$ be two weighting vectors, $U \in \mathcal{U}_{\mathrm{i}}^{1 / n}$ and let $\hat{v}_{\boldsymbol{p}, \boldsymbol{w}}^{U}$ be the capacity on $N$ associated to $\boldsymbol{p}, \boldsymbol{w}$ and $U$. Then, for any nonempty subset $A$ of $N$, we get

$$
\min \left(\sum_{i \in A} p_{i}, \sum_{i=1}^{|A|} w_{i}\right) \leq \hat{v}_{\boldsymbol{p}, \boldsymbol{w}}^{U}(A) \leq \max \left(\sum_{i \in A} p_{i}, \sum_{i=1}^{|A|} w_{i}\right) .
$$

Proof. Given a nonempty subset $A$ of $N$, by Proposition 6 we have

$$
\hat{v}_{\boldsymbol{p}, \boldsymbol{w}}^{U}(A) \geq v_{\boldsymbol{p}, \boldsymbol{w}}^{U}(A) \geq \min \left(\sum_{i \in A} p_{i}, \sum_{i=1}^{|A|} w_{i}\right) .
$$

The second inequality is obtained as follows:

$$
\begin{aligned}
\hat{v}_{p, w}^{U}(A) & =\max _{B \subseteq A} v_{p, w}^{U}(B) \leq \max _{B \subseteq A} \max \left(\sum_{i \in B} p_{i}, \sum_{i=1}^{|B|} w_{i}\right) \\
& \leq \max \left(\sum_{i \in A} p_{i}, \sum_{i=1}^{|A|} w_{i}\right) .
\end{aligned}
$$

Corollary 2. Let $\boldsymbol{p}$ and $\boldsymbol{w}$ be two weighting vectors, $U \in \mathcal{U}_{\mathrm{i}}^{1 / n}$ and let $v_{\boldsymbol{p}, \boldsymbol{w}}^{U}$ and $\hat{v}_{\boldsymbol{p}, \boldsymbol{w}}^{U}$ be the game and the capacity on $N$ associated to $\boldsymbol{p}, \boldsymbol{w}$ and $U$, respectively. Then, for any nonempty subset $A$ of $N$, we have

$$
\sum_{i \in A} p_{i}=\sum_{i=1}^{|A|} w_{i} \Rightarrow \hat{v}_{\boldsymbol{p}, \boldsymbol{w}}^{U}(A)=v_{\boldsymbol{p}, \boldsymbol{w}}^{U}(A)=\sum_{i \in A} p_{i} .
$$

Proof. It is obvious from Proposition 6 and Corollary 1.

\section{SUOWA operators}

We now introduce SUOWA operators as Choquet integral-based operators with respect to the capacities $\hat{v}_{p, w}^{U}$.

Definition 12. Let $\boldsymbol{p}$ and $\boldsymbol{w}$ be two weighting vectors and let $U \in \widetilde{\mathcal{U}}^{1 / n}$. The semi-uninorm based ordered weighted averaging (SUOWA) operator associated to $\boldsymbol{p}, \boldsymbol{w}$ and $U$ is the function $S_{p, w}^{U}: \mathbb{R}^{n} \longrightarrow \mathbb{R}$ given by

$$
S_{p, w}^{U}(\boldsymbol{x})=\sum_{i=1}^{n} s_{i} x_{[i]},
$$

where $s_{i}=\hat{v}_{\boldsymbol{p}, \boldsymbol{w}}^{U}\left(A_{[i]}\right)-\hat{v}_{\boldsymbol{p}, \boldsymbol{w}}^{U}\left(A_{[i-1]}\right)$ for all $i \in N, \hat{v}_{\boldsymbol{p}, \boldsymbol{w}}^{U}$ is the capacity associated to $\boldsymbol{p}, \boldsymbol{w}$ and $U$, $A_{[i]}=\{[1], \ldots,[i]\}$ and, by convention, $A_{[0]}=\varnothing$. 
According to Remark 1, and since $\hat{v}_{\boldsymbol{p}, \boldsymbol{w}}^{U}$ are normalized capacities, SUOWA operators are continuous, monotonic, idempotent, compensative and homogeneous of degree 1 functions. In addition to this, SUOWA operators satisfy a very interesting property when we consider idempotent semi-uninorms. Given $\boldsymbol{x} \in \mathbb{R}^{n}$, each value $x_{[i]}$ is associated to the weights $p_{[i]}$ and $w_{i}$. If both weights are equal for all $i \in N$, then the corresponding weights of the SUOWA operator coincide with them and, consequently, the value provided by the SUOWA operator is the same as the value returned by the weighted mean and the OWA operator (in fact, as we can see from the proof of Proposition 7, given $i \in N$, the value $s_{[i]}$ coincides with $p_{[i]}$ and $w_{i}$ when $p_{[i]}=w_{i}$ and $\left.\sum_{j=1}^{i-1} p_{[j]}=\sum_{j=1}^{i-1} w_{j}\right)$. Notice that, in general, WOWA operators do not satisfy this property.

Proposition 7. Let $\boldsymbol{p}$ and $\boldsymbol{w}$ be two weighting vectors, $U \in \mathcal{U}_{\mathrm{i}}^{1 / n}$ and let $\boldsymbol{x} \in \mathbb{R}^{n}$ such that $p_{[i]}=w_{i}$ for all $i \in N$. Then,

$$
S_{p, w}^{U}(\boldsymbol{x})=M_{p}(\boldsymbol{x})=O_{w}(\boldsymbol{x}) .
$$

Proof. Let $\boldsymbol{x} \in \mathbb{R}^{n}$ such that $p_{[i]}=w_{i}$ for all $i \in N$. Then, for all $i \in N$, we have

$$
\sum_{j \in A_{[i]}} p_{j}=\sum_{j=1}^{i} p_{[j]}=\sum_{j=1}^{i} w_{j}=\sum_{j=1}^{\left|A_{[i]}\right|} w_{j},
$$

and, by Corollary 2, we get

$$
\hat{v}_{p, w}^{U}\left(A_{[i]}\right)=\sum_{j=1}^{i} p_{[j]}
$$

Therefore, for all $i \in N$,

$$
s_{i}=\hat{v}_{\boldsymbol{p}, \boldsymbol{w}}^{U}\left(A_{[i]}\right)-\hat{v}_{\boldsymbol{p}, \boldsymbol{w}}^{U}\left(A_{[i-1]}\right)=\sum_{j=1}^{i} p_{[j]}-\sum_{j=1}^{i-1} p_{[j]}=p_{[i]},
$$

and, consequently,

$$
S_{p, w}^{U}(\boldsymbol{x})=\sum_{i=1}^{n} p_{[i]} x_{[i]}=M_{p}(\boldsymbol{x})=O_{w}(\boldsymbol{x}) .
$$

It is also worth noting that SUOWA operators preserve the order of the corresponding semiuninorms.

Proposition 8. Let $\boldsymbol{p}$ and $\boldsymbol{w}$ be two weighting vectors, and $U_{1}, U_{2} \in \widetilde{\mathcal{U}}^{1 / n}$. If $U_{1}(x, y) \leq U_{2}(x, y)$ for all $(x, y) \in[0,1]^{2}$, then

$$
S_{p, w}^{U_{1}}(\boldsymbol{x}) \leq S_{p, w}^{U_{2}}(\boldsymbol{x})
$$

for all $\boldsymbol{x} \in \mathbb{R}^{n}$.

Proof. Given $U \in \widetilde{\mathcal{U}}^{1 / n}$ and $\boldsymbol{x} \in \mathbb{R}^{n}$, since $S_{p, \boldsymbol{w}}^{U}(\boldsymbol{x})$ can be written as

$$
S_{p, \boldsymbol{w}}^{U}(\boldsymbol{x})=\sum_{i=1}^{n} \hat{v}_{\boldsymbol{p}, \boldsymbol{w}}^{U}\left(A_{[i]}\right)\left(x_{[i]}-x_{[i+1]}\right)
$$


it is sufficient to prove that $\hat{v}_{p, w}^{U_{1}}(A) \leq \hat{v}_{p, w}^{U_{2}}(A)$ for all $A \subseteq N$. As $U_{1}(x, y) \leq U_{2}(x, y)$ for all $(x, y) \in[0,1]^{2}$, we have $v_{p, w}^{U_{1}}(A) \leq v_{p, w}^{U_{2}}(A)$ for all $A \subseteq N$ and, by Proposition 3 , the monotonic cover of the games also satisfy the corresponding inequalities.

Taking into account the smallest and the largest (idempotent) semi-uninorms, we have immediately the following result.

Corollary 3. Let $\boldsymbol{p}$ and $\boldsymbol{w}$ be two weighting vectors. Then the following holds:

1. If $U \in \widetilde{\mathcal{U}}^{1 / n}$, then $S_{p, w}^{U_{\perp}}(\boldsymbol{x}) \leq S_{p, w}^{U}(\boldsymbol{x}) \leq S_{p, \boldsymbol{w}}^{U_{\top}}(\boldsymbol{x})$ for all $\boldsymbol{x} \in \mathbb{R}^{n}$.

2. If $U \in \mathcal{U}_{\mathrm{i}}^{1 / n}$, then $S_{p, w}^{U_{\min }}(\boldsymbol{x}) \leq S_{p, w}^{U}(\boldsymbol{x}) \leq S_{p, w}^{U_{\max }}(\boldsymbol{x})$ for all $\boldsymbol{x} \in \mathbb{R}^{n}$.

Proof. Obvious from Proposition 8.

\section{Examples}

Two examples are provided in this section. The first one illustrates the construction of games and capacities associated to three different idempotent semi-uninorms. In the second one we show that, although WOWA operators are also a particular case of Choquet integral (see Torra [25]), SUOWA and WOWA operators are different classes of aggregation operators. To do this, we will use an example given by Llamazares [14].

Example 3. Consider the weighting vectors $\boldsymbol{p}=(0.6,0.2,0.1,0.1)$ and $\boldsymbol{w}=(0.4,0,0,0.6)$. Besides $U_{\min }$ and $U_{\max }$, we are also going to use $U_{\text {am }}$, the idempotent semi-uninorm obtained by means of the arithmetic mean:

$$
U_{\mathrm{am}}(x, y)=\left\{\begin{array}{l}
\min (x, y) \text { if }(x, y) \in[0,0.25]^{2}, \\
\max (x, y) \text { if }(x, y) \in[0.25,1]^{2} \backslash\{(0.25,0.25)\}, \\
(x+y) / 2 \text { otherwise. }
\end{array}\right.
$$

In Table 1 we show the games and the capacities associated to these idempotent semi-uninorms. It is worth noting that the values of the capacities can be obtained by using the recursive expression

$$
\hat{v}(A)=\max \left(v(A), \max _{i \in A} \hat{v}(A \backslash\{i\})\right) .
$$

For instance, the value $\hat{v}_{\boldsymbol{p}, \boldsymbol{w}}^{U_{\min }}(\{1,2,3\})$ can be obtained as

$$
\begin{aligned}
\hat{v}_{\boldsymbol{p}, \boldsymbol{w}}^{U_{\min }}(\{1,2,3\}) & =\max \left(v_{\boldsymbol{p}, \boldsymbol{w}}^{U_{\min }}(\{1,2,3\}), \hat{v}_{\boldsymbol{p}, \boldsymbol{w}}^{U_{\min }}(\{1,2\}), \hat{v}_{\boldsymbol{p}, \boldsymbol{w}}^{U_{\min }}(\{1,3\}), \hat{v}_{\boldsymbol{p}, \boldsymbol{w}}^{U_{\min }}(\{2,3\})\right) \\
& =\max (0.4,0.6,0.6,0.3)=0.6
\end{aligned}
$$


Table 1: Games and capacities associated to $U_{\min }, U_{\mathrm{am}}$ and $U_{\max }$

\begin{tabular}{lccllll} 
& \multicolumn{2}{c}{$U_{\min }$} & \multicolumn{2}{c}{$U_{\mathrm{am}}$} & \multicolumn{2}{c}{$U_{\max }$} \\
\hline \multicolumn{1}{c}{ Set } & $v_{\boldsymbol{p}, \boldsymbol{w}}^{U_{\min }}$ & $\hat{v}_{\boldsymbol{p}, \boldsymbol{w}}^{U_{\min }}$ & $v_{\boldsymbol{p}, \boldsymbol{w}}^{U_{\mathrm{am}}}$ & $\hat{v}_{\boldsymbol{p}, \boldsymbol{w}}^{U_{\mathrm{am}}}$ & $v_{\boldsymbol{p}, \boldsymbol{w}}^{U_{\max }}$ & $\hat{v}_{\boldsymbol{p}, \boldsymbol{w}}^{U_{\max }}$ \\
\hline$\{1\}$ & 0.6 & 0.6 & 0.6 & 0.6 & 0.6 & 0.6 \\
$\{2\}$ & 0.2 & 0.2 & 0.3 & 0.3 & 0.4 & 0.4 \\
$\{3\}$ & 0.1 & 0.1 & 0.25 & 0.25 & 0.4 & 0.4 \\
$\{4\}$ & 0.1 & 0.1 & 0.25 & 0.25 & 0.4 & 0.4 \\
$\{1,2\}$ & 0.4 & 0.6 & 0.6 & 0.6 & 0.8 & 0.8 \\
$\{1,3\}$ & 0.4 & 0.6 & 0.55 & 0.6 & 0.7 & 0.7 \\
$\{1,4\}$ & 0.4 & 0.6 & 0.55 & 0.6 & 0.7 & 0.7 \\
$\{2,3\}$ & 0.3 & 0.3 & 0.3 & 0.3 & 0.3 & 0.4 \\
$\{2,4\}$ & 0.3 & 0.3 & 0.3 & 0.3 & 0.3 & 0.4 \\
$\{3,4\}$ & 0.2 & 0.2 & 0.2 & 0.25 & 0.2 & 0.4 \\
$\{1,2,3\}$ & 0.4 & 0.6 & 0.65 & 0.65 & 0.9 & 0.9 \\
$\{1,2,4\}$ & 0.4 & 0.6 & 0.65 & 0.65 & 0.9 & 0.9 \\
$\{1,3,4\}$ & 0.4 & 0.6 & 0.6 & 0.6 & 0.8 & 0.8 \\
$\{2,3,4\}$ & 0.4 & 0.4 & 0.4 & 0.4 & 0.4 & 0.4 \\
$N$ & 1 & 1 & 1 & 1 & 1 & 1 \\
\hline
\end{tabular}

Example 4. Let us consider the weighting vectors $\boldsymbol{p}=(0.5,0.25,0.2,0.05)$ and $\boldsymbol{w}=(0,0.5,0.5,0)$, and $\boldsymbol{x}=(9,10,5,4)$ (see Llamazares [14]). In the case of WOWA operators, it is easy to check that, whatever the quantifier used, the value of the WOWA operator associated to $p$ and $\boldsymbol{w}$ is

$$
W_{p, w}(9,10,5,4)=0 \cdot 10+1 \cdot 9+0 \cdot 5+0 \cdot 4=9 .
$$

Now we calculate the capacities associated to the semi-uninorms $U_{\perp}$ and $U_{\mathrm{T}}$ (both functions are showed in Figure 3). Notice that in this case, the capacities coincide with the games (that is, the games are monotonic), and they are given in Table 2.

In both cases, the weights of the SUOWA operator are

$$
\begin{array}{ll}
s_{1}=\hat{v}_{p, \boldsymbol{w}}^{U}(\{2\}) & =0, \\
s_{2}=\hat{v}_{\boldsymbol{p}, \boldsymbol{w}}^{U}(\{2,1\})-\hat{v}_{\boldsymbol{p}, \boldsymbol{w}}^{U}(\{2\}) & =0.75, \\
s_{3}=\hat{v}_{\boldsymbol{p}, \boldsymbol{w}}^{U}(\{2,1,3\})-\hat{v}_{\boldsymbol{p}, \boldsymbol{w}}^{U}(\{2,1\}) & =0.25, \\
s_{4}=\hat{v}_{\boldsymbol{p}, \boldsymbol{w}}^{U}(N)-\hat{v}_{\boldsymbol{p}, \boldsymbol{w}}^{U}(\{2,1,3\}) & =0,
\end{array}
$$



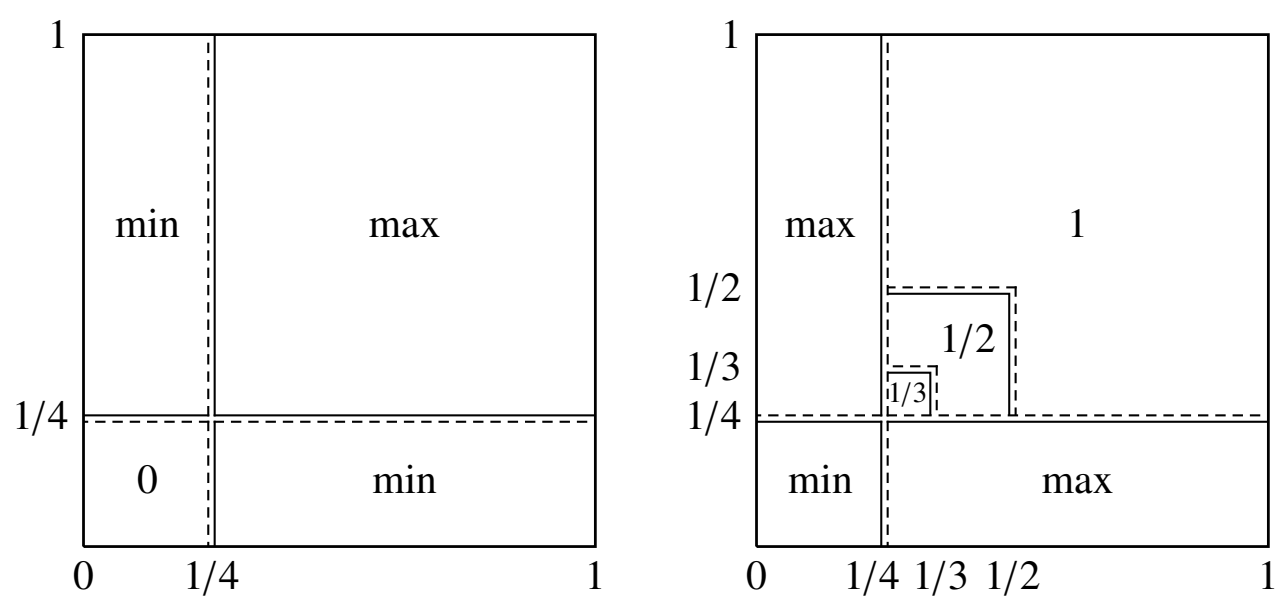

Figure 3: Representation of $U_{\perp}$ and $U_{\mathrm{T}}$ when $n=4$.

Table 2: Capacities associated to $U_{\perp}$ and $U_{\mathrm{T}}$

\begin{tabular}{lll}
\hline \multicolumn{1}{c}{ Set } & $\hat{v}_{p, w}^{U_{\perp}}$ & $\hat{v}_{p, w}^{U_{\top}}$ \\
\hline$\{1\}$ & 0 & 0.5 \\
$\{2\}$ & 0 & 0 \\
$\{3\}$ & 0 & 0 \\
$\{4\}$ & 0 & 0 \\
$\{1,2\}$ & 0.75 & 0.75 \\
$\{1,3\}$ & 0.7 & 0.7 \\
$\{1,4\}$ & 0.55 & 0.55 \\
$\{2,3\}$ & 0.45 & 0.45 \\
$\{2,4\}$ & 0.3 & 0.3 \\
$\{3,4\}$ & 0.25 & 0.25 \\
$\{1,2,3\}$ & 1 & 1 \\
$\{1,2,4\}$ & 1 & 1 \\
$\{1,3,4\}$ & 1 & 1 \\
$\{2,3,4\}$ & 0.5 & 1 \\
$N$ & 1 & 1 \\
\hline
\end{tabular}


where $U \in\left\{U_{\perp}, U_{T}\right\}$. Therefore,

$$
\begin{aligned}
S_{p, w}^{U_{\perp}}(9,10,5,4) & =S_{p, w}^{U_{\top}}(9,10,5,4) \\
& =0 \cdot 10+0.75 \cdot 9+0.25 \cdot 5+0 \cdot 4=8 .
\end{aligned}
$$

According to (1) of Corollary 3, given any $U \in \widetilde{\mathcal{U}}^{1 / n}$, we have

$$
S_{p, w}^{U}(9,10,5,4)=8
$$

Consequently, SUOWA and WOWA operators are different classes of aggregation operators.

It is worth noting that WOWA operators are defined by considering quantifiers generating the weighting vector $\boldsymbol{w}$ (see Torra and Godo [36]), where the quantifier is the identity when $\boldsymbol{w}=\boldsymbol{\eta}$; that is, in order to get the weighted mean we need to use a specific quantifier. But in the case of SUOWA operators, any semi-uninorm belonging to the set $\widetilde{\mathcal{U}}^{1 / n}$ allows us to obtain the weighted mean.

Notice also that in Example 4, it seems that SUOWA operators have a better performance than WOWA operators. On the one hand, it is easy to check that the values returned by the weighted mean and the OWA operator are $M_{p}(9,10,5,4)=8.2$ and $O_{w}(9,10,5,4)=7$. Therefore, the value returned by SUOWA operators, 8 , seems more consistent than the value given by WOWA operators, 9. On the other hand, WOWA operators only take into account the value provided by the first information source and assign a weight of zero to the value provided by the third information source, $x_{3}=5$, even if this value is associated with the weights $p_{3}=0.2$ and $w_{3}=0.5$. In the case of SUOWA operators, the weight assigned to $x_{3}=5$ is 0.25 . Obviously, this behavior happens in this numerical example and no conclusions can be deduced for the general case. So, the comparison between the behavior of WOWA and SUOWA operators deserves a more in-depth study.

\section{Conclusion}

In some practical cases it is necessary to aggregate values by using two weighting vectors. The first one provides information about the reliability of each information source while the second one allows to weight the values according to their ordering. Although there exist in the literature a large number of aggregation operators, WOWA operators seem to be the most suitable for dealing with this kind of situations (see Llamazares [14]). As WOWA operators, the functions introduced in this paper are Choquet integral-based operators with respect to normalized capacities. Therefore, SUOWA operators are continuous, monotonic, idempotent, compensative and homogeneous of degree 1 functions, and, consequently, they constitute an alternative to WOWA operators to deal with this kind of aggregation problems.

\section{Acknowledgment}

The author is grateful to two anonymous referees for valuable suggestions and comments. Part of this research was done during a stay of the author at the University of Trento (Italy). I 
would like to thank all members of MEDIA group for the warm hospitality and especially Ricardo Alberto Marques Pereira for helpful comments and suggestions. The partial financial support from the University of Valladolid, the Spanish Ministry of Economy and Competitiveness (Project ECO2012-32178) and the Junta de Castilla y León (Consejería de Educación, Project VA066U13) is gratefully acknowledged.

\section{References}

[1] R. R. Yager, On ordered weighted averaging operators in multicriteria decision making, IEEE Trans. Syst., Man, Cybern. 18 (1988) 183-190.

[2] V. Torra, The weighted OWA operator, Int. J. Intell. Syst. 12 (1997) 153-166.

[3] V. Torra, L. Godo, Continuous WOWA operators with application to defuzzification, in: T. Calvo, G. Mayor, R. Mesiar (Eds.), Aggregation Operators: New Trends and Applications, volume 97 of Studies in Fuzziness and Soft Computing, Physica-Verlag, Heidelberg, 2002, pp. 159-176.

[4] V. Torra, Y. Narukawa, Modeling Decisions: Information Fusion and Aggregation Operators, Springer, Berlin, 2007.

[5] R. R. Yager, N. Alajlan, A generalized framework for mean aggregation: Toward the modeling of cognitive aspects, Inform. Fusion 17 (2014) 65-73.

[6] R. R. Yager, Quantifier guided aggregation using OWA operators, Int. J. Intell. Syst. 11 (1996) 49-73.

[7] R. R. Yager, Including importances in OWA aggregation using fuzzy systems modelling, IEEE Trans. Fuzzy Syst. 6 (1998) 286-294.

[8] T. Calvo, R. Mesiar, Criteria importances in median-like aggregation, IEEE Trans. Fuzzy Syst. 9 (2001) $662-$ 666.

[9] K. J. Engemann, D. P. Filev, R. R. Yager, Modelling decision making using immediate probabilities, Int. J. Gen. Syst. 24 (1996) 281-294.

[10] Z. S. Xu, Q. L. Da, An overview of operators for aggregating information, Int. J. Intell. Syst. 18 (2003) $953-969$.

[11] J. M. Merigó, Fuzzy decision making with immediate probabilities, Comput. Ind. Eng. 58 (2010) 651-657.

[12] J. Lin, Y. Jiang, Some hybrid weighted averaging operators and their application to decision making, Inform. Fusion 16 (2014) 18-28.

[13] W. Wang, Comment on "Some hybrid weighted averaging operators and their application to decision making", Inform. Fusion 16 (2014) 84-85.

[14] B. Llamazares, An analysis of some functions that generalizes weighted means and OWA operators, Int. J. Intell. Syst. 28 (2013) 380-393.

[15] X. Liu, Preference solutions of probability decision making with RIM quantifiers, Int. J. Intell. Syst. 20 (2005) 1253-1271.

[16] J. Lin, Reply to the comment on "Some hybrid weighted averaging operators and their application to decision making", Inform. Fusion 16 (2014) 86-88.

[17] R. R. Yager, A. Rybalov, Uninorm aggregation operators, Fuzzy Sets Syst. 80 (1996) 111-120.

[18] M. Grabisch, T. Murofushi, M. Sugeno (Eds.), Fuzzy Measures and Integrals: Theory and Applications, volume 40 of Studies in Fuzziness and Soft Computing, Physica-Verlag, Heidelberg, 2000.

[19] M. Grabisch, C. Labreuche, A decade of application of the Choquet and Sugeno integrals in multi-criteria decision aid, Ann. Oper. Res. 175 (2010) 247-286.

[20] M. Sugeno, Theory of Fuzzy Integrals and its Applications, Phd thesis, Tokyo Institute of Technology, 1974.

[21] G. Choquet, Theory of capacities, Ann. Inst. Fourier 5 (1953) 131-295.

[22] T. Murofushi, M. Sugeno, A theory of fuzzy measures. Representation, the Choquet integral and null sets, J. Math. Anal. Appl. 159 (1991) 532-549.

[23] D. Denneberg, Non-Additive Measures and Integral, Kluwer Academic Publisher, Dordrecht, 1994.

[24] M. Grabisch, J. Marichal, R. Mesiar, E. Pap, Aggregation Functions, Cambridge University Press, Cambridge, 2009.

[25] V. Torra, On some relationships between the WOWA operator and the Choquet integral, in: Proceedings of the 
Seventh International Conference on Information Processing and Management of Uncertainty in KnowledgeBased Systems (IPMU'98), Paris (France), pp. 818-824.

[26] J. Fodor, J.-L. Marichal, M. Roubens, Characterization of the ordered weighted averaging operators, IEEE Trans. Fuzzy Syst. 3 (1995) 236-240.

[27] M. Grabisch, Fuzzy integral in multicriteria decision making, Fuzzy Sets Syst. 69 (1995) 279-298.

[28] M. Grabisch, On equivalence classes of fuzzy connectives - the case of fuzzy integrals, IEEE Trans. Fuzzy Syst. 3 (1995) 96-109.

[29] J. C. Fodor, R. R. Yager, A. Rybalov, Structure of uninorms, Int. J. Uncertain. Fuzziness Knowl.-Based Syst. 5 (1997) 411-427.

[30] J. Fodor, B. De Baets, Uninorm basics, in: P. P. Wang, D. Ruan, E. E. Kerre (Eds.), Fuzzy Logic: A Spectrum of Theoretical \& Practical Issues, volume 215 of Studies in Fuzziness and Soft Computing, Springer, Berlin, 2007, pp. 49-64.

[31] H.-W. Liu, Semi-uninorms and implications on a complete lattice, Fuzzy Sets Syst. 191 (2012) 72-82.

[32] B. De Baets, Idempotent uninorms, Eur. J. Oper. Res. 118 (1999) 631-642.

[33] M. Maschler, B. Peleg, The structure of the kernel of a cooperative game, SIAM J. Appl. Math. 15 (1967) 569-604.

[34] M. Maschler, B. Peleg, L. S. Shapley, The kernel and bargaining set for convex games, Int. J. Game Theory 1 (1971) 73-93.

[35] T. Calvo, A. Kolesárová, M. Komorníková, R. Mesiar, Aggregation operators: properties, classes and construction methods, in: T. Calvo, G. Mayor, R. Mesiar (Eds.), Aggregation Operators: New Trends and Applications, volume 97 of Studies in Fuzziness and Soft Computing, Physica-Verlag, Heidelberg, 2002, pp. 3-104.

[36] V. Torra, L. Godo, Averaging continuous distributions with the WOWA operator, in: Proceedings of the Second European Workshop on Fuzzy Decision Analysis and Neural Networks for Management, Planning and Optimization (EFDAN'97), Dortmund (Germany), pp. 10-19. 